\title{
JobChain: An Integrated Blockchain Model for Managing Job Recruitment for Ministries in Sultanate of Oman
}

\author{
Vinu Sherimon ${ }^{1}$, Sherimon P.C ${ }^{2}$, Alaa Ismaeel ${ }^{3}$ \\ Department of Information Technology, Higher College of Technology, Muscat, Sultanate of Oman ${ }^{1}$ \\ Faculty of Computer Studies, Arab Open University, Muscat, Sultanate of Oman ${ }^{2,3}$ \\ Faculty of Science, Minia University, Minya, Egypt ${ }^{3}$
}

\begin{abstract}
Industries around the world has revolutionized with the arrival of blockchain technology. Blockchain applications and use cases are in the process of development in different domains. This research presents a blockchain platform "JobChain" to manage the job recruitments. The case study is conducted for the job recruitments in various Ministries in Sultanate of Oman. Currently, in Oman, citizens are aware of the job vacancies through the advertisements posted in newspapers or social media. A job seeker then applies for the desired job and thereafter the qualified candidates are called for tests/ interviews. To ease this process, a solution based on blockchain which includes various Ministries and the citizens/ residents of Sultanate of Oman is proposed in this research. Ministries can post the job vacancies in the blockchain and qualified citizen(s) can submit their application. Relevant cryptographic functions are used to verify the authenticity of the participants in the blockchain network. The citizens feel the existence of a trusted secure government, which is mandatory for the development of a country. Unlike traditional models, blockchain eliminates the need of intermediary agents (e.g. Job Consultancies) thereby providing direct communication between the participants of the blockchain. The proposed blockchain framework helps the citizens in Oman to get updated about the job vacancies. Hyperledger Composer Playground is used to design and test the proposed blockchain business network. Preliminary results show that the participants and assets are created successfully and the transactions to approve a job vacancy and a job application is done through the proposed blockchain network.
\end{abstract}

Keywords-Blockchain; permissioned; chaincode; hyperledger composer playground; job recruitment

\section{INTRODUCTION}

As part of the Industrial Revolution 4.0, blockchain technologies are accelerating at a great velocity. It was first developed for bitcoin cryptocurrency [1]. Blockchain technology runs on distributed networks. The goal of this technology was to provide a global environment which is decentralized, transparent and secure to conduct transactions [1]. They have the potential to transform and reshape data governance. In centralized database systems, the system management, access control, protection against cyber-attacks, etc. are done by a single entity. But in blockchain, every node in the network updates the data independently. The transactions are kept with every participant in an openly distributed ledger securely and it is immutable. Data once added to the blockchain cannot be repudiated or updated. Also, the users can cross-check the validity of the data provided in the blockchain. The need for a third-party or a single entity control is eliminated here [1]. This technology offers digital trust between the participants involved in the network. Cryptographic algorithms will ensure the authenticity of the participants and the transactions will be carried out only by the authorized participants [2].

A blockchain system is classified into two different types of networks: Permissionless blockchains and permissioned blockchains. Ethereum and Bitcoin are examples of Permissionless blockchain. In this network, there is no central authority and any node can join the network to submit a transaction. These transactions are then validated by miner nodes, who receives incentives on calculating a complex hash value that results in a new block creation. The incentives (usually a bitcoin) encourage the miner nodes to stay in the network and they compete among themselves in mining a new block. On the other hand, in permissioned blockchain, a central authority decides about the members of a network. Only authenticated and authorized participants can join the network.

In Sultanate of Oman, Ministry of Civil Services (MOCS) is responsible for recruiting the citizens to the various ministries in Oman. The traditional method was to collect the requirements from different Ministries through Human Resources Management System (HRMS) and place notifications in the newspapers. Job seekers then apply for the desired job and thereafter the qualified candidates are called for tests/ interviews. Since it was very time-consuming, later SMS (Short Messaging Service) was introduced so that the job seeker can send an SMS with the desired job code and the employee code registered in Ministry of Manpower (MOM). MOM validates the applicant's data and other relevant documents. Then the suitable candidates go through aptitude tests and interviews.

In the existing system, the following are the drawbacks:

- The Job vacancy notifications are published in newspapers, which costs money.

- The Job seeker should remember the job codes to send SMS to MOCS. 
- If a Job seeker wishes to update his CV, for example, to add a new qualification, he/she must submit the new certificates to MOM, and wait for validation, which is time consuming and difficult.

To enhance the existing recruitment process, we propose a blockchain based platform "JobChain" to ease the recruitment process.

Blockchains are digital decentralized ledgers. Blockchain applications builds trust, accountability and transparency among the participants in the network. A permissioned private blockchain [2] which requires the verification of the participants is proposed in this project. Every participant who is involved in the blockchain requires permission to execute transactions on the blockchain. Membership services authenticates and authorizes the identity of the participants. The blockchain creates a ledger which stores the whole history of blockchain transactions which are immutable, and it is distributed throughout the blockchain network. Each node in the blockchain maintains its own copy of the ledger. Whenever an update or delete transaction is initiated it is done based on the consensus between the nodes. The blockchain creates more transparency between the job seeker and the ministries. Here every job vacancy is posted in the blockchain by the concerned Ministry. MOCS approves/ rejects the posted job vacancy.

The rest of the paper is organized as follows: Section 2 presents the related work. Section 3 describes Permissioned blockchain networks and the different components. The architecture of the proposed model is shown in Section 4. Section 5 presents the implementation details of the model. Section 6 presents the results and Section 7 describes the significance of the proposed model followed by conclusion and future in Section 8.

\section{ANALYSIS OF RELATED WORK}

Blockchain technology is disrupting different industries in many countries now. Estonia is the first country to test the blockchain technology in 2008, even before the white paper on bitcoin was published [3]. At that time, since the term "blockchain" was not in use, the technology was named as "hash-linked time-stamped". The blockchain enabled eEstonia project, integrates all government services to a single platform. The country had developed a blockchain technology KSI, which secures e-services such as e-Healthcare records, eJudiciary, e-Government, e-Land registry, e-Banking, etc. by storing the data in a distributed ledger [3]. This avoids data misuse and corruptions. A web-based blockchain voting program (e-Voting) was used in 2017 in Estonia [3]. Using this system, a citizen can cast his/her vote and he/she can also verify the data (voting choice) received by the government [3].

The first government endorsed blockchain platform is launched in Dubai on October 30, 2018. It is as part of the vision to make the Emirati government paperless by 2021 [4]. It integrates the digitized services to the lives of the normal citizens. Various blockchain applications in different sectors such as education, healthcare, energy, etc. are also launched in Dubai, the pioneer in blockchain technology.

In an urge to ensure the food safety and authenticity, in 2017, Dubai has launched 'Food Watch', a digital platform that digitize the food safety and nutritional information of all foods served in the country [5]. Initially, all establishments which handle high-risk foods are required to update the platform with the information related to the foods they handle. This enables the consumers and the food quality checkers to verify the authenticity of food. The future phase of this project includes to integrate blockchain, big data and IOT technologies to track food products from farm to table [5].

UAE Banks Federation has initiated the process of implementing blockchain to digitize various processes in bank. For example, adopting blockchain technology in KYC (Know Your Customer) processes to enhance the experience of customers [6]. The Swiss city of Zug pilot tested "e-voting pilot", a blockchain application to cast votes. In 2017, an Ethereum-based application "uPort" was launched in Zug to digitize ID's of residents. This ID was used by the residents to cast votes using the blockchain application [7].

A private blockchain based project was introduced in the Republic of Georgia to register land titles thus becoming the first government to introduce blockchain in this domain [8]. The application allows citizens to validate their land registrations securely. The project is in the process of expansion by including services such as purchase and sale of land titles, demolition of lands, mortgages, etc. [8].

Kuwait has introduced its first blockchain application for email which ensures secured transactions [9]. As part of Vision 2030, Saudi Arabia also has partnered up with IBM to implement several blockchain applications in various sectors [10]. Bahrain's economic development board (EDB) and Abu Dhabi global market (ADGM) have agreed to collaborate on blockchain based developments and digital payments [11]. In Sultanate of Oman, Blockchain Solutions \& Services Co (BSS) is the pioneer in conducting many forums and workshops about Blockchain [12]. BSS is working with different entities to establish the needed infrastructure to implement this new powerful technology [12].

As per the literature review, no research has been reported regarding the application of blockchain in job recruitments.

\section{PERMissioned BlockCHAIN}

Permissioned blockchains can be utilized by such business organizations where the participants are known to each other $[\mathrm{ABB}+18]$. They are used in cases where there exists partial trust or no trust between the participants. Here, a central authority is authorized to decide on the rules for the participants to join the network and submit transactions. Transactions are validated by every participant in the blockchain by reaching a Consensus. They are executed by a Smart Contract, which updates the data in a distributed ledger and adds a new block to blockchain.

Consensus-Because of the distributed nature of blockchain, every participant should agree on certain rules to validate the block generated and to add it in the blockchain. These rules are called Consensus and they work together with Smart Contracts to guarantee the order, correctness and validity of transactions. There are different types of consensus algorithms based on several classifications. For instance, if the classification is based on the method of block selection, then voting based 
algorithms and lottery-based algorithms are the two types. In voting type, majority of the participants validate the blocks or transactions and consensus is achieved fast, whereas in lotterybased systems, the winner of the process proposes a new block and adds it to the blockchain.

Apache Kafka is a voting-based consensus algorithm. If the classification is based on the type of peers, there are two types of algorithms-competing peers and non-competing peers. In competing peers' algorithm, the peers compete on the next block to be appended on the blockchain whereas in noncompeting peers' algorithm, only few peers work on block creation, and the rest of the peers validates the block. Some other consensus algorithms depend on the architecture of execution of the transactions. In the Order-Execute model, the ordering of the transaction takes place before the execution of transactions. Proof-of-Work (PoW) algorithm is an example of this type $[\mathrm{ABB}+18]$. In Execute-Order-Validate model, the transactions are executed initially, followed by ordering of the executed transactions, and the validation of the outputs. The transaction execution model of Hyperledger Fabric is ExecuteOrder-Validate style.

Membership Service Provider (MSP) - Every trusted member of a blockchain network must have a digital identity/ certificate to allow access to resources and to submit transactions. Each of these identities must be issued by a trusted authority. An MSP is a component that determines the valid identities of an organization. The default MSP implementation uses X.509 certificates issued by Certificate Authority (CA). In blockchain networks, sometimes there may be more than one CAs. Also, the Root CA issues certificates to various Intermediate CAs, which in turn issues certificates to members of different organizations. In this way, a chain of trust is established. In a blockchain network, MSP identifies the trusted Root CAs and Intermediate CAs (if any) which are used to define the members of a domain. MSP can also determine specific member roles and define the access privileges of members in the network as per their roles.

Chaincodes - The business logic of the blockchain network is defined in Chaincodes. It forms the heart of the blockchain network. In a Permissionless blockchain like Ethereum, chain codes are referred as Smart Contracts. Also, both the terms, Smart Contract and Chaincode are used interchangeably in Permissioned Blockchains. A Chaincode is installed on all nodes of the blockchain network. The client application invokes the Chaincode with the support of APIs and submit requests for transactions. Also, the transactions can be executed by itself upon meeting certain criteria. After the transactions are validated by the network participants, the results are updated in the shared distributed ledger by all the participants of the network.

Authorization Policy - It defines the policies for the set of nodes who will execute the Chaincode and validates its results. The policies take the form of "MSP.Role". If the policy is defined as Org1.admin, then the policy designates the admin of Org1 MSP.

Channels - This is required if a large business network includes a subset of private networks which do not want to share some confidential information with the rest of the network. An organization can participate in multiple blockchain networks via separate channels. All the participants in a channel share information and coordinate with each other according to the authorization policy.

Ledger - There are two different components for a ledger world state and a blockchain. The world state holds the current value of an object and the blockchain keeps the history of all the transactions. When a new transaction is successfully committed, the world state gets updated with the new results. A key-value pair represents a world state. The blockchain consists of blocks, connected with each other and each block includes a set of transactions.

Data Storage - The world state can be implemented in two ways - using CouchDB or LevelDB. LevelDB supports simple key-value pairs and CouchDB supports complex queries.

\section{JobChain ARChiteCTURE}

Fig. 1 shows the architecture of the proposed blockchain application.

Ministries can post the job vacancies in the blockchain, which will be approved by the Ministry of Civil Services. As per the requirements, qualified citizen(s) can submit their application. This will be validated by the Ministry of Manpower. If the job application is initially approved, the concerned Ministry will inform the candidates about the exam/ interview details. Also, the results of the exam/ interview will be uploaded in the blockchain and the status of job vacancies are updated accordingly.

Every blockchain network consists of participants, assets, and transactions. The participant Ministry represents different ministries in Sultanate of Oman. MinistryStaff represents the staff under each ministry who posts the categories of jobs and the current vacancies. Here MOCS (Ministry of Civil Services) is responsible to approve the job vacancies posted by other ministries. Job Seeker's role is to view the job vacancies and to apply for the job. MOM (Ministry of Manpower) is responsible to validate the job seeker's data.

The asset Job Categories represent the categories of jobs posted by the ministries. Job Vacancies signify the current vacancies in the ministries and Job Application characterizes the application of job seekers.

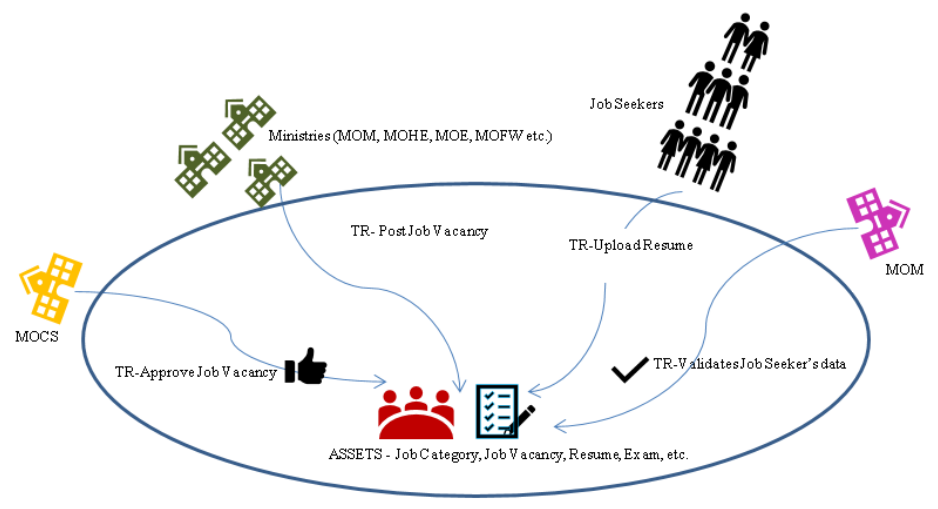

Fig. 1. Proposed Framework. 
Post Job Vacancy transaction is used by Ministries to post the vacancies. Approve Job Vacancy is used by MOCS to approve the vacancies posted by other ministries. ValidatesJobSeeker's data is used by MOM to validate the data of a Job Seeker.

\section{MODEL IMPLEMENTATION}

An object-oriented modeling language is used to define the domain model for a business network definition. All the resources such as assets, participants, transactions, and events are defined in the model file.

Every Ministry participant is identified by an Id, followed by name and address. Here address is defined as a concept. It consists of multiple information combined in a single concept.

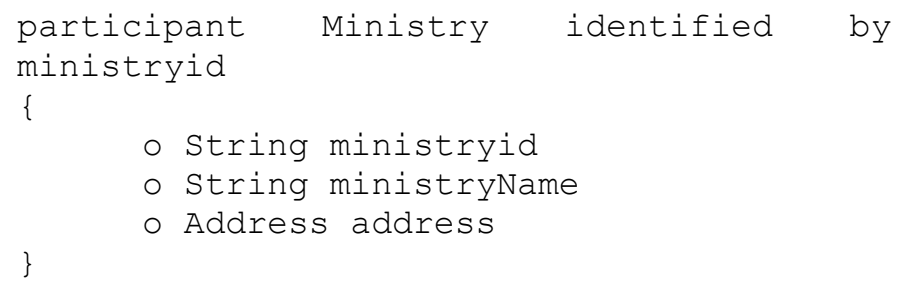

An abstract object called JobChainParticipant is defined and the participants MinistryStaff and JobSeeker are extended from this abstract object as they both share common properties.

abstract participant JobChainParticipant identified by participantId

\{

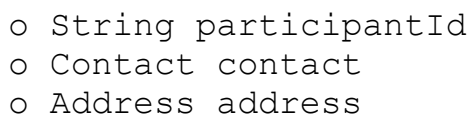

The MinistryStaff participant has additional attributes such as department, jobTitle, userStatus and a relationship to Ministry object. userStatus is defined as an enumeration to define the status of the user.

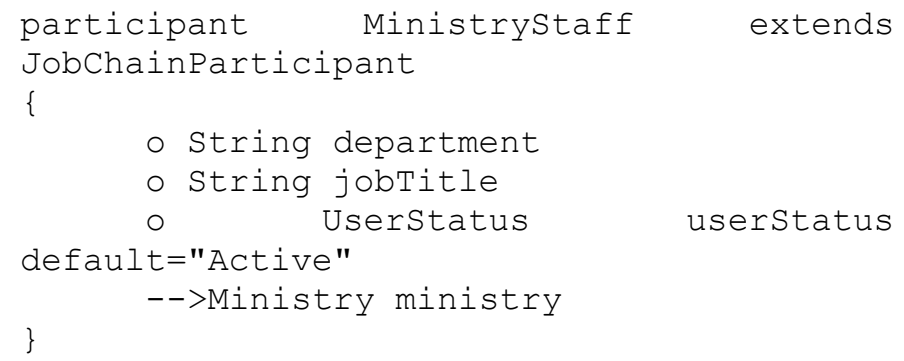

The assets of the blockchain are JobCategory, JobVacancy, etc. The asset JobCategory is given below:

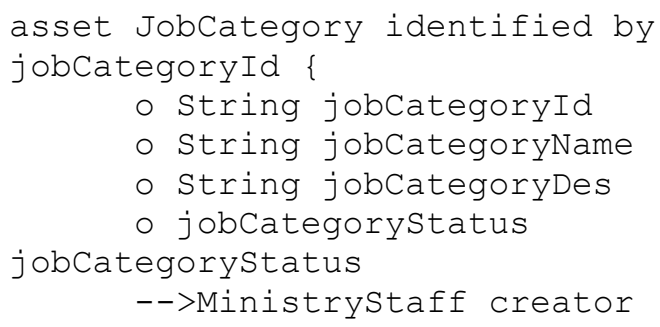

In addition to the attributes, jobCategoryId, jobCategoryName, jobCategoryDes, and jobCategoryStatus, a relationship exists to MinistryStaff object in the above asset.

Similarly, different transactions such as CreateMinistry, CreateMinistryStaff, PostJobCategory, PostJobVacancy, etc. are also defined in the model.

To execute the different transactions defined in the model file, a logic file should be implemented in the blockchain network. This file contains the codes required to implement each of these transactions. Corresponding to each transaction, a function is written in the logic file, which is used to update the attributes of the participants/ assets. These updates are saved to the participant and asset registries.

\section{Testing AND Results}

Hyperledger composer playground, an open source web application tool is used to develop the JobChain prototype. Hyperledger is a project of open source Blockchain and distributed ledger technology by the Linux Foundation. This tool is used to build the business model, test the model and deploy the network to runtime. It keeps the blockchain model in the browser storage.

\section{A. Creation of Participants}

Initially, the participant registry is empty. The admin submits the transaction CreateMinistry in the blockchain and register two members of the Ministry participant in the blockchain.

Ministry of Health - MOH (id: 100), who can post a Job Category and Job Vacancy.

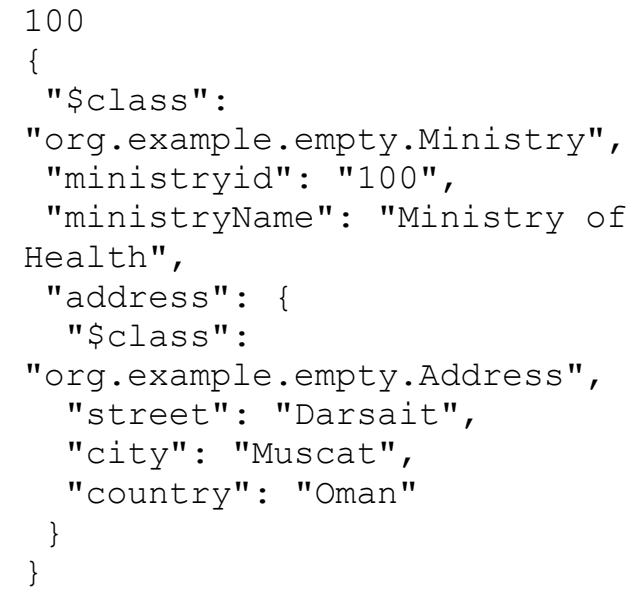

Ministry of Civil Service - MOCS (id: 102) who can post a Job Category and Job Vacancy and can approve/ reject the Job Vacancies posted by other ministries.

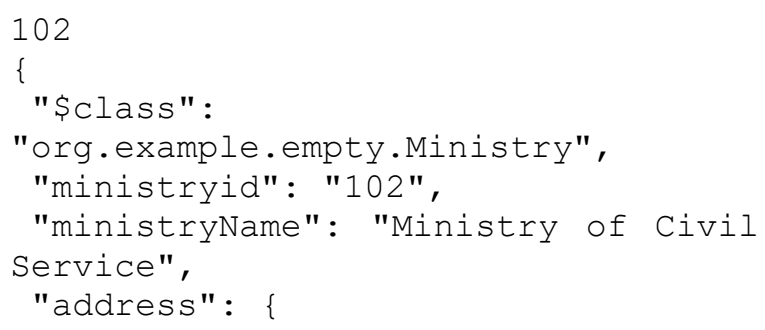




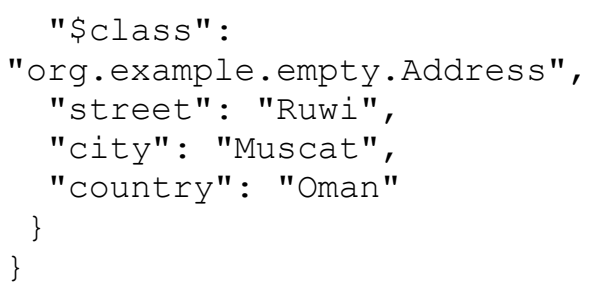

Later, $\mathrm{MOH}$ submits the transaction CreateMinistrystaff to register an $\mathrm{MOH}$ staff in the blockchain. For example, the data of a $\mathrm{MOH}$ staff (id: 1) is registered as shown below:

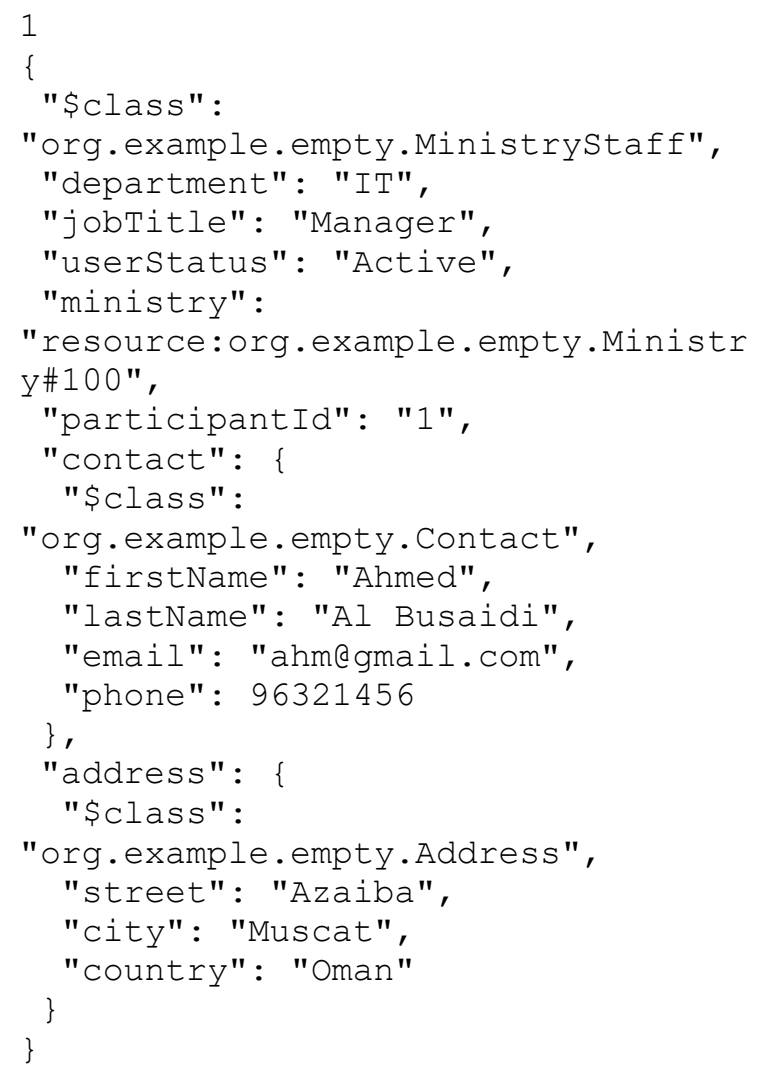

A job seeker can register in the blockchain through CreateJobseeker transaction (id: 0738).

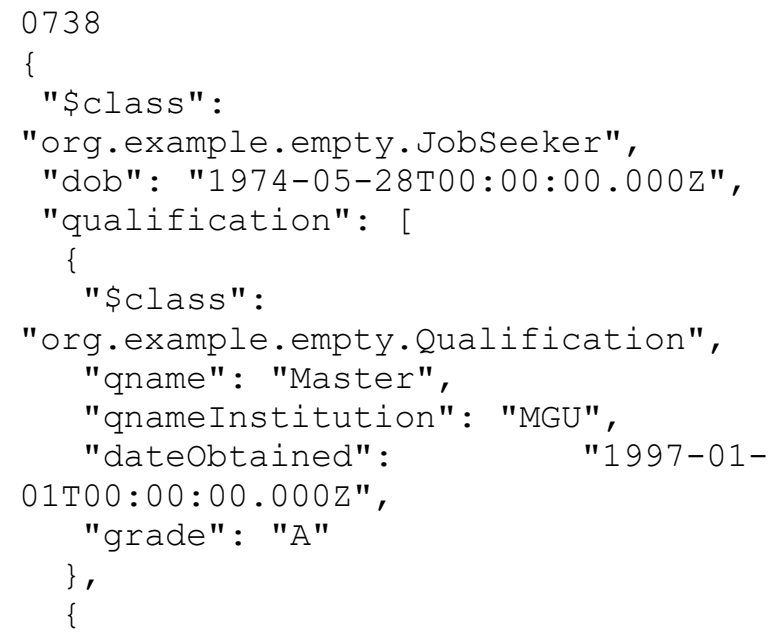

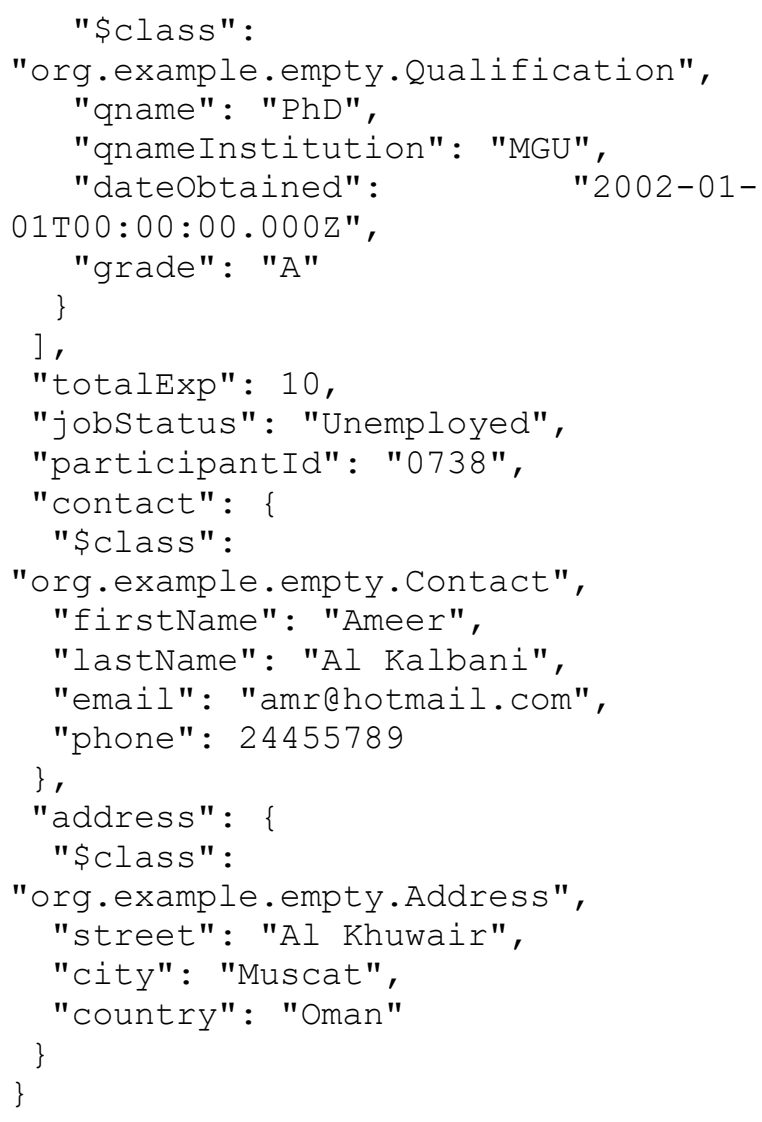

\section{B. Creation of Assets}

Initially, the asset registry is empty. Now, assume that $\mathrm{MOH}$ would like to register a JobCategory asset in the blockchain. The staff of $\mathrm{MOH}$ submits the transaction PostJobCategory and adds an instance of the asset JobCategory (id: C1) in the asset registry.

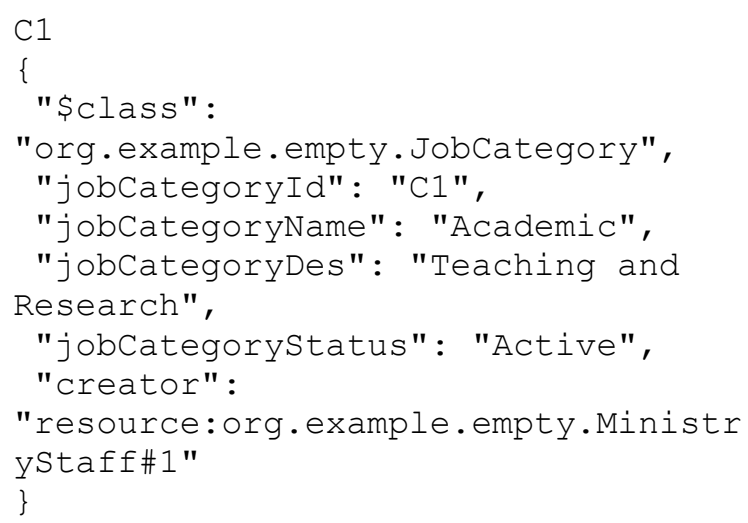

After the category $\mathrm{C} 1$ is registered in the blockchain, an instance of the asset JobVacancy under $\mathrm{C} 1$ is also posted by MOH by submitting PostJobVacancy transaction.

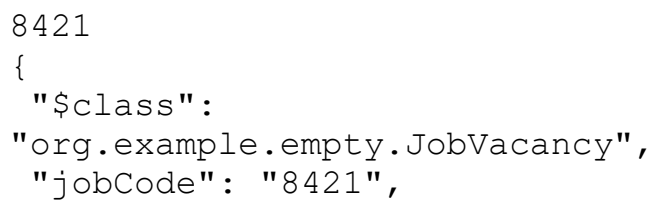




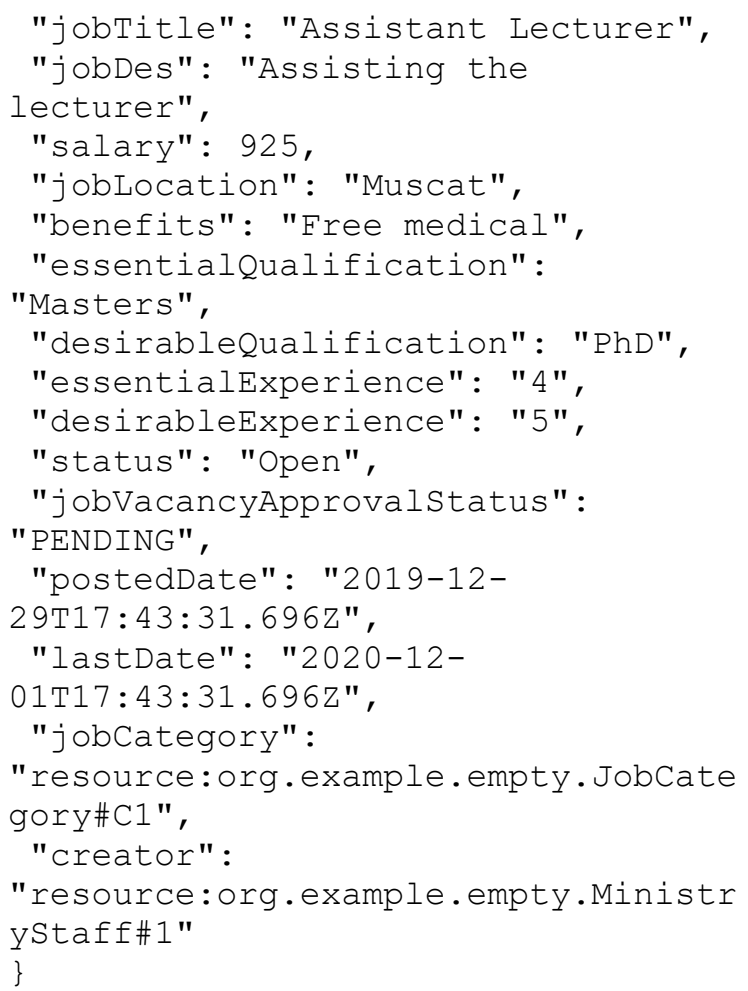

Next, assume that the JobSeeker Ammer Al Kalbani (id: 0738) wish to apply for the job 8421 given above. This is done by submitting the transaction PostJobApplication into the blockchain.

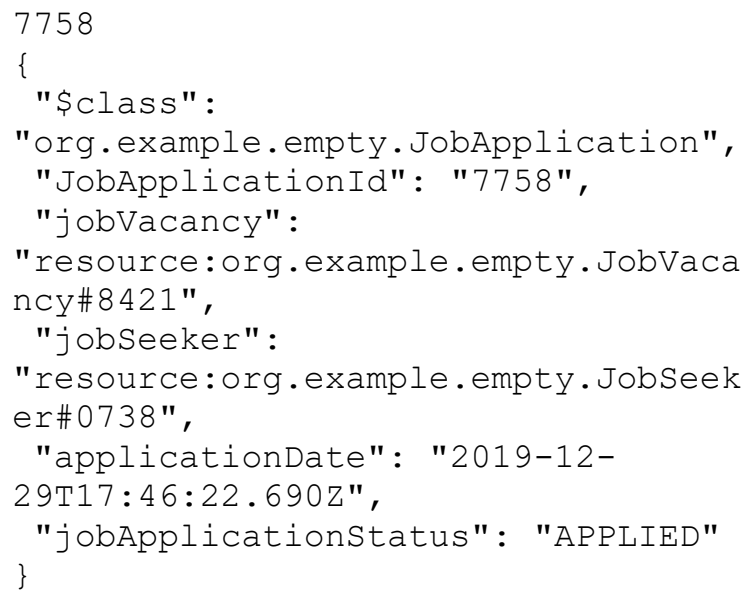

\section{Approval of Job Vacancy}

Now, MOCS is given the rights to update the JobVacancy asset. This is done through the ApproveJobVacancy transaction. The attribute jobVacancyApprovalStatus is set to the value APPROVED, once it is approved by MOCS.

For instance, MOCS submits the transaction ApproveJobVacancy to approve the JobVacancy (id: 8421). This results in the change in the attribute value of jobVacancyApprovalStatus to APPROVED in the asset JobVacancy (Fig. 2).

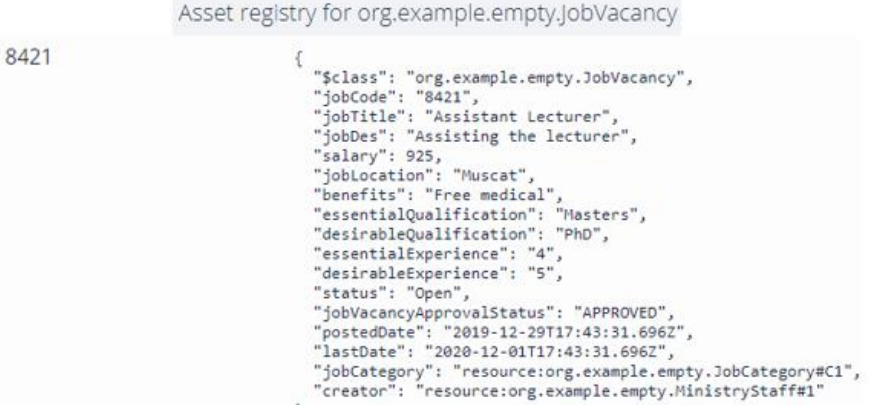

Fig. 2. Job Vacancy Asset.

\section{Approval of Job Application}

When a job seeker registers a job application, the current status of the job application is APPLIED. The concerned Ministry is responsible to check the JobApplication and update its status. If the initial requirements are met, it is changed to APPROVED by the concerned ministry Otherwise the status is changed to REJECTED.

For instance, $\mathrm{MOH}$ checks the application submitted against its Job vacancy. Since the initial requirement are met, MOH submits the transaction ApproveJobApplication, which approves the JobApplication (id: 7758) submitted by the JobSeeker (id: 0738). The asset registry given in Fig. 3 shows the change in jobApplicationStatus.

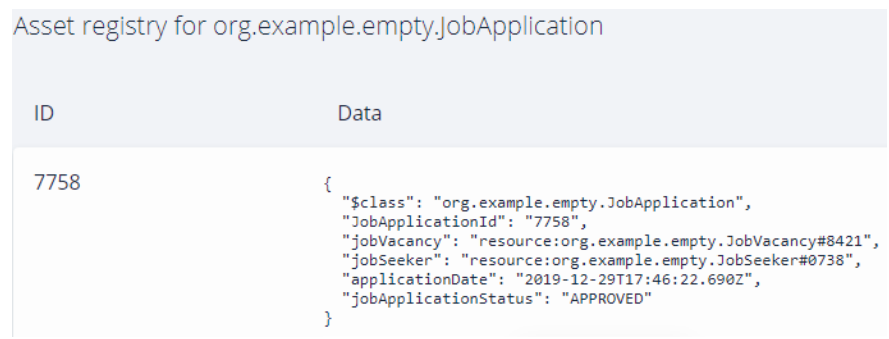

Fig. 3. Job Application Asset.

\section{SIGNIFICANCE OF JOBCHAIN}

A blockchain network is a form of distributed ledger design, which is spread across multiple nodes where each node maintains an identical copy of the ledger. The main feature of this technology is that there is no central authority to administer the data. It is updated independently and ensure data synchronization. The data is secured using cryptographic techniques. The records generated are real-time, so each node keeps an up-to-date copy of the data or transactions [13]. The transaction records created are permanent and immutable [13].

The proposed research will impact the country in many ways. A common platform exists where the Ministries collaborate with each other [14]. Citizens often find difficulty to understand the category of jobs, the nature of jobs, current vacancies, etc. Also, currently the job vacancies are advertised in the newspapers and the job seeker should remember the job code and send SMS to apply for the job. Distributed Ledger Technology eliminates the need for intermediaries in any transactions. Through the proposed blockchain framework, the citizens can directly view the job vacancies in each Ministry. If 
the job profile matches with his/ her education and experience, the citizen can apply for the job.

\section{CONCLUSION AND FUTURE WORK}

Blockchain applications offer several unique features. A blockchain is not owned by a single entity; it is the asset of all the participants in the blockchain network. Data stored in a blockchain is secured against deletion/alteration. The stakeholders of a blockchain have a common platform to collaborate with each other. As every participant owns a copy of the blockchain data, it is resilient against any attacks. Also, every participant can verify the correctness of the transactions. We have presented a blockchain architecture to manage the job recruitments in various Ministries in Sultanate of Oman. The proposed model will enhance the trust, validity and accountability between Job Seekers and Ministries in the Sultanate. The full implementation of the proposed framework in Hyperledger Composer Playground is planned as the future scope of this paper.

\section{ACKNOWLEDGMENT}

The research leading to these results has received funding from the Research Council (TRC) of the Sultanate of Oman under the Block Funding Program BFP/ RGP/ ICT/ 18/ 114.

\section{REFERENCES}

[1] Yli-Huumo, J., Ko, D., Choi, S., Park, S., \& Smolander, K. (2016). Where is current research on blockchain technology? —a systematic review. PloS one, 11(10), e0163477.

[2] Greenspan, G. (2015). MultiChain private blockchain-White paper. URl: Retrieved from http://www. multichain. com/download/Multi Chain-White-Paper. pdf.
[3] Drechsler, W. (2018). Pathfinder: e-Estonia as the $\beta$-version. JeDEMeJournal of eDemocracy and Open Government, 10(2), 1-22.

[4] Emirates Blockchain Strategy 2021. Retrieved from https://government. ae/en/about-the-uae/strategies-initiatives-and-awards/federal-governm ents-strategies-and-plans/emirates-blockchain-strategy-2021.

[5] Darin Detwiler. (2018, Feb 27). Retrieved from https://www.ibm. com/blogs/blockchain/2018/02/one-nations-move-to-increase-food-safet y-with-blockchain/.

[6] UBF's CEOs Advisory Council explored adoption of Blockchain in banks. Retrieved from https://www.unlock-bc.com/news/2018-1217/ubfs-ceos-advisory-council-explored-adoption-of-blockchain-inbanks.

[7] Wofie Zhao. (2018, June 11). Swiss City Plans Blockchain Voting Pilot Using Ethereum-Based IDs. Retrieved from https://www.coindesk.com/ swiss-city-plans-to-vote-on-blockchain-using-ethereum-digital-id.

[8] Laura Shin. (2017, Feb 7). Retrieved from https://www.forbes .com/sites/laurashin/2017/02/07/the-first-government-to-secure-landtitles-on-the-bitcoin-blockchain-expands-project/\#3a7e53d04dcd.

[9] Prepare for Blockchain Disruption. Retrieved from https://www. protiviti.com/KW-en/node/94216.

[10] Saudi Arabia Partners With IBM To Use Blockchain for Better Government Services. Retrieved from https://ethereumworldnews .com/saudi-arabia-ibm-blockchain-services/.

[11] Arabian Business Industries. Retrieved from https://www.arabianb usiness.com/technology/403858-bahrain-minister-lauds-blockchain-astrue-mark-of-progress.

[12] MuscatDaily.Com. Retrieved from https://www.muscatdaily.com /Archive/Business/Blockchain-infrastructure-to-be-rolled-out-soon-inOman-58s7.

[13] Michael, JW, Alan Cohn, and Jared R. Butcher. "Blockchain technology." The Journal (2018).

[14] Peck, M. "Reinforcing the Links of the Blockchain." IEEE Future Directions Initiative White Paper (2017). 